\title{
Adaptación y validación del inventario de autotrascendencia para adultos en el contexto argentino
}

\section{Adaptation and Validation of the Self Transcendence}

Inventory for Adults in the Argentine context

Adaptação e validação do inventário de autotranscendência para adultos no contexto argentino

\section{Hugo Simkin ${ }^{1}$}

Recibido: 26 de septiembre de 2018

Aprobado: 21 de mayo de 2019

Publicado: 21 de junio de 2019

Cómo citar este artículo:

Simkin. H. (2019). Adaptación y validación del inventario de autotrascendencia para adultos en el contexto argentino. Pensando Psicología, 15(25), 1-15.

DOI: https://doi.org/10.16925/2382-3984.2019.01.05

Artículo de investigación. https://doi.org/10.16925/2382-3984.2019.01.05

1 Universidad de Buenos Aires

ORCID: https://orcid.org/0000-0001-7162-146X

Correo electrónico: hugosimkin@psi.uba.ar 


\title{
Resumen
}

Introducción: En los últimos años el estudio de la autotrascendencia ha cobrado un creciente interés para la psicología. En particular, el Inventario de Evaluación para Adultos (ASTI) constituye una de las principales técnicas disponibles para su evaluación empírica.

Objetivo: El presente trabajo se propone adaptar y validar la escala al contexto argentino en una muestra de 205 estudiantes de nivel medio y superior de la Ciudad Autónoma de Buenos Aires.

Método: Se analizó la estructura interna del instrumento mediante la aplicación de un análisis factorial confirmatorio verificando empíricamente el modelo de dos dimensiones, aportando así evidencias de validez de constructo a la técnica en el contexto argentino.

Palabras clave: adaptación, análisis factorial, autotrascendencia, espiritualidad, validación.

\begin{abstract}
Introduction: In recent years self-transcendence has gained increasing interest within psychological literature. Currently, the Adult Self Transcendence Inventory (ASTI) is one of the main scales used in empirical research for measuring self transcendence.

Objective: The present study aims to adapt and validate the scale to the Argentine context in a sample of 205 high school and university students in the Autonomous City of Buenos Aires.

Method: A confirmatory factor analysis of the factor structure of the adapted Adult Self Transcendence Inventory was conducted to assess whether the scale's purported 2 factors emerged. The results generally supported the 2-factor model.
\end{abstract}

Keywords: adaptation, confirmatory factorial analysis, self transcendence, spirituality, validation

\section{Resumo}

Introdução: Nos últimos anos, o estudo da autotranscendência tem despertado crescente interesse pela psicologia. Em particular, o Inventário de Avaliação de Adultos (ASTI) é uma das principais técnicas disponíveis para avaliação empírica.

Objetivo: 0 presente trabalho propõe adaptar e validar a escala para o contexto argentino em uma amostra de 205 estudantes de nível médio e superior da Cidade Autônoma de Buenos Aires.

Método: A estrutura interna do instrumento foi analisada através da aplicação de uma análise fatorial confirmatória, verificando empiricamente o modelo bidimensional, evidenciando a validade de constructo da técnica no contexto argentino.

Palavras-chave: adaptação, análise fatorial confirmatória, autotranscendência, espiritualidade, validação. 


\section{Introduction}

El interés por explorar distintas experiencias vinculadas a la autotrascendencia, la espiritualidad o la religiosidad ha promovido la construcción de numerosas escalas de evaluación psicológica que contribuyen a su indagación empírica (Kapuscinski y Masters, 2010; Simkin, 2017b). En el contexto latinoamericano se han identificado distintas adaptaciones locales de técnicas frecuentemente empleadas en psicología, entre las que se destacan la Age Universal I-E Scale (Allport y Ross, 1967; RiveraLedesma, Zavala-Jimenez, Montero-Lopez Lena, y Maria Garcia-Mendez, 2016; Simkin y Etchezahar, 2013), la Escala de Afrontamiento Religioso o Religious Coping Scale (RCOPE) (González-Rivera y Pagán Torres, 2018; Mezzadra y Simkin, 2017; Pargament, Smith, Koenig, y Perez, 1998), la Escala de Creencias Post-Criticas o Post-Critical Belief scale (PCBS) (Duriez, Fontaine, Hutsebaut, y Leuven, 2000; Rabbia, Brussino, y Vaggione, 2012) o la Escala de Evaluación de Espiritualidad y Sentimientos Religiosos o Assessment of Spirituality and Religious Sentiments (ASPIRES) (Piedmont, 2004; Simkin, 2017a; Simkin y Piedmont, 2018). En el presente, una de las técnicas que ha cobrado mayor relevancia para la evaluación de la autotrascendencia en la literatura especializada es el Inventario de Autotrascendencia para Adultos o Adult Self Transcendence Inventory (ASTI) (Levenson, Jennings, Aldwin, y Shiraishi, 2005). Sin embargo, no se han relevado estudios en el contexto argentino que procuren adaptar y validar la escala al ámbito local. Por este motivo, el presente artículo se propone realizar una adaptación de la técnica que contribuya a la indagación del fenómeno en ámbito local.

\section{El Inventario de Autotrascendencia para Adultos (ASTI)}

La autotrascendencia suele definirse como la superación de los límites del yo individual, generalmente en relación con la percepción de conexión a otras personas, tanto en un plano secular como espiritual (Levenson et al., 2005; Piedmont, 1999, 2004; Reed, 1991). De acuerdo con García-Romeu (2010) una de las técnicas de evaluación de la autotrascendencia más relevantes en la literatura especializada es el Inventario de Autotrascendencia para Adultos o Adult Self Transcendence Inventory (ASTI) (Levenson et al., 2005). La técnica incluye 18 ítems que se responden en una escala tipo Likert con cinco anclajes de respuesta en función del grado de acuerdo de los participantes. La ASTI distingue dos facetas: 1) autotrascendencia; y 2) alienación, presentando en su construcción original una consistencia interna aceptable tanto para el primer factor $(a=0,75)$ como para el segundo $(a=0,64)$. El inventario ha sido empleado en distintos países como Alemania (Koller, Levenson, y Glück, 
2017; Konig y Gluck, 2014), Australia (Ireland, 2013), Austria (Glück et al., 2013; Koller et al., 2017; Konig y Gluck, 2014; König y Glück, 2013), Canadá (Beaumont, 2009; Ireland, 2013), Corea (Lee, Choun, Aldwin, y Levenson, 2015), Eslovaquia (Roháriková, Špajdel, Cviková, y Jagla, 2013), Estados Unidos (Ai, Wink, Gall, Dillon, y Tice, 2017; Choi y Landeros, 2011; Greene, Britton, y Shepherd, 2016; Jennings, Aldwin, Levenson, Spiro, y Mroczek, 2006; Le, 2011; Le y Levenson, 2005; Morledge et al., 2013; Vieten et al., 2014), Francia (Michael Dambrun, 2017; Michaël Dambrun y Ricard, 2012), Reino Unido (Ireland, 2013), y en población coreana (Kim y Knight, 2015) y vietnamita (Le, 2008) residente en los Estados Unidos. Para la construcción de la técnica, Levenson et al., (2005) retoman la noción de Tornstam (1994) de gerotrascendencia, relativa al desarrollo de la autotrascendencia hacia el final de la vida. Sin embargo, si bien los estudios iniciales se centran en la tercera edad (Rajani, 2015), Levenson et al., (2005) amplían el rango etario de la escala para relevar el constructo también en adultos jóvenes. De manera similar, en el presente artículo, en atención a la necesidad de explorar el fenómeno con una mayor amplitud (Boyatzis, 2009; Levenson, Aldwin, y Igarashi, 2013) se ha optado por incluir, además de población adulta, población adolescente.

En los estudios de validación, tal como ha sido sugerido por la literatura (Heo, Kim, y Faith, 2015), la consistencia interna de las dimensiones de la ASTI fue estimada a partir del estadístico alfa de Cronbach, arrojando resultados aceptables $(0,67>a>$ 0,83). El estudio exploratorio realizado por Levenson et al., (2005) empleando rotación Varimax contribuyó a identificar dos dimensiones claramente diferenciadas entre sí que integran los 18 ítems que componen ASTI. La estructura factorial de la ASTI posteriormente fue evaluada en la literatura empleando ecuaciones estructurales, esto permitió observar que los índices de ajuste considerados indican que el modelo de dos factores de la ASTI resulta adecuado para los datos recabados en campo (Koller et al., 2017; Lee et al., 2015). En el mismo sentido, el RMSEA suele presentar valores inferiores a .05 (Lee et al., 2015), guarismos considerados aceptables para la literatura especializada (Hair, Black, Babin, y Anderson, 2009). Además, los índices de ajuste (CFI) presentan regularmente valores superiores a 0,90, acorde a lo que se considera recomendable (Bentler, 1990; Hu y Bentler, 1999; Lee et al., 2015). Por su parte, la distribución $\chi 2$ y sus grados de libertad resultan aceptables, informándose en su mayoría valores inferiores a 4 (Jackson, Gillaspy, y Purc-Stephenson, 2009; Lee et al., 2015). 


\section{Método}

\section{Participantes}

La selección de la muestra fue de tipo intencional y estuvo compuesta por 205 estudiantes de nivel medio y superior de la Ciudad Autónoma de Buenos Aires con edades comprendidas entre 13 y 30 años $(M=18,01$; $D E=3,94)$ y de ambos sexos (hombres $=49,3 \%$; mujeres $=50,7 \%$ ).

\section{Instrumentos \\ Inventario de Autotrascendencia para Adultos o Adult Self Transcendence Inventory (ASTI)}

\section{(Levenson et al., 2005)}

ASTI es un cuestionario autoadministrable que incluye 18 ítems que evalúan dos dimensiones: 1) autotrascendencia (Self Transcendence [ST]) (ítems 1, 2, 3, 5, 6, 7, 11, 12, 13, 15, 16, y 18); y 2) alienación (Alientation [A]) (ítems 4, 8, 9, 10, 14 y 17). De acuerdo con la literatura, las propiedades psicométricas de ASTI han resultado adecuadas en el contexto internacional (Koller et al., 2017; Lee et al., 2015; Levenson et al., 2005). La escala presenta un formato de respuesta tipo Likert con cinco anclajes de respuesta en función del grado de acuerdo de los participantes siendo: 1 "completamente de acuerdo" y 5 "completamente en desacuerdo". La adaptación al español fue realizada siguiendo los estándares metodológicos internacionales que sugiere la International Test Commission (ITC) para la adaptación correcta de las técnicas de evaluación entre contexto idiomáticos diversos (Muñiz, Elosua, y Hambleton, 2013).

\section{Cuestionario de datos sociodemográficos}

Se elaboró un cuestionario ad hoc que solicitaba a los participantes consignar la edad y el género.

\section{Procedimiento}

Los sujetos fueron invitados a participar del estudio de forma voluntaria, solicitándoles debidamente su consentimiento. Antes de responder a la técnica de evaluación se les hizo constar que los datos derivados de esta investigación se utilizarían con fines 
exclusivamente científicos bajo la Ley Nacional 25326 de protección de los datos personales. A lo largo de la administración de los cuestionarios se permaneció en presencia de los participantes.

\section{Análisis de datos}

Para el análisis estadístico se emplearon los programas SPSS 22 y EQS 6.1. En primer lugar, se analizó la confiabilidad de ASTI a partir del análisis de consistencia interna, empleando el estadístico Alfa Ordinal (Dominguez-Lara, 2018) y el coeficiente Omega de McDonald (Zhang y Yuan, 2016; Zinbarg, Revelle, Yovel, y Li, 2005). En segundo lugar, se evaluó la validez de constructo en la realización de un análisis factorial confirmatorio (AFC), empleando el método GLS robusto, siguiendo recomendaciones previas (Schermelleh-Engel, Moosbrugger, y Müller, 2003). El modelo fue evaluado mediante los Índices De Bondad De Ajuste $\chi^{2}$, Índice De Ajuste Incremental o Incremental Fit Index (IFI), el Índice de Ajuste Normado o Normed Fit Index (NFI), el Índice de Ajuste No Normado o Non-Normed Fit Index (NNFI), el Índice de Ajuste Comparado o Comparative fit index (CFI) y el Error de Aproximación Cuadrático Medio o Root Mean Square Error of Approximation (RMSEA).

\section{Resultados}

Debido a las recientes recomendaciones de la literatura, se empleó el coeficiente alfa ordinal para la evaluación de la consistencia interna (Dominguez-Lara, 2018; Espinoza y Novoa-Muñoz, 2018; Ventura, 2018) tanto para el factor autotrascendencia $(a=0,71)$, como para el factor alienación $(a=0,50)$. Por su parte, el coeficiente Omega de McDonald (Ventura León y Caycho Rodriguez, 2017; Viladrich, Angulo-Brunet, y Doval, 2017) arrojó asimismo guarismos aceptables tanto para el factor autostrascendencia $(\omega=0,96)$ como para el factor alienación $(\omega=0,93)$.

Posteriormente, se procedió a realizar el análisis factorial confirmatorio, evaluado a partir de los Índices De Bondad De Ajuste $\chi^{2}$, IFI, NFI, NNFI, CFI y el RMSEA, tal como se ha sugerido en los antecedentes (Schumacker y Lomax, 2004). Este análisis permitió comprobar que el modelo propuesto se ajusta a los datos recabados en campo (Hair et al., 2009).

En la tabla 1 se puede observar que los índices IFI, NFI, NNFI y CFI alcanzaron guarismos superiores a 0,90 y RMSEA de 0,08, valores sugeridos como puntos de corte que permiten distinguir un buen ajuste de uno regular (Schreiber, Nora, Stage, Barlow, y King, 2006). 
Tabla 1. Análisis Factorial Confirmatorio de la Escala ASTI.

\begin{tabular}{ccccccc}
\hline & $\boldsymbol{X}_{(\boldsymbol{g l})}$ & $\boldsymbol{N F I}$ & $\boldsymbol{N N F I}$ & $\boldsymbol{C F I}$ & $\boldsymbol{I F I}$ & $\boldsymbol{R M S E A}($ IC) \\
\hline ASTI & $856,896_{(134)}$ & 0,938 & 0,956 & 0,961 & 0,962 & $0,087(0,076 ; 0,099)$ \\
\hline
\end{tabular}

${ }^{\star}$ Modelo bidimensional.

Fuente: elaboración propia

A continuación, en la figura 1 puede observarse el modelo del Inventario de Autotrascendencia para Adultos.

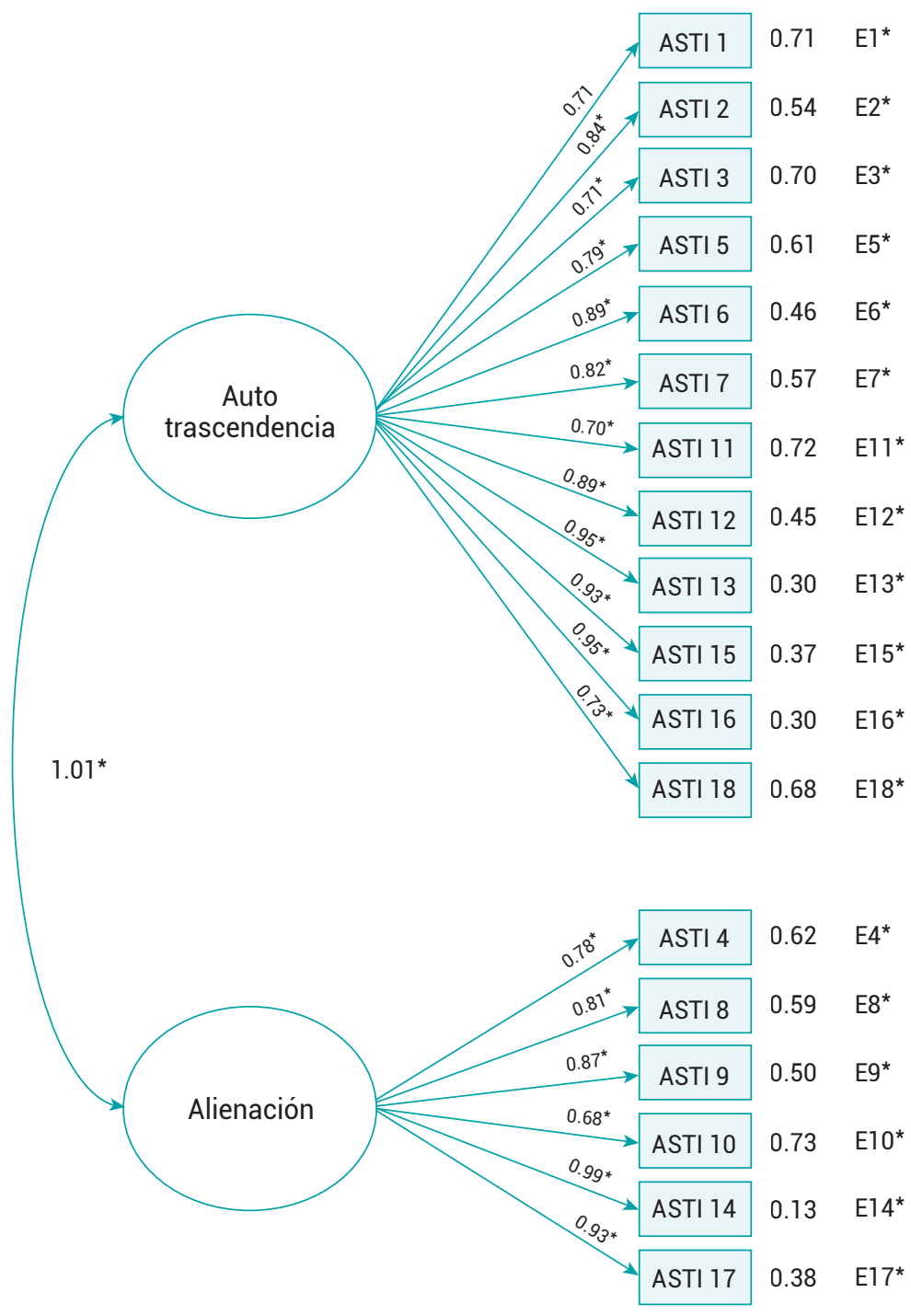

Figura 1. Modelo estructural del Inventario de Autotrascendencia para Adultos. Fuente: elaboración propia 
Con excepción del ítem 10, los parámetros estimados estandarizados fueron significativos $(p<0,05)$ (Jackson et al., 2009) y se encuentran por arriba de los valores óptimos $(r>0,70)$ lo que implica que presentan un coeficiente de determinación que estaría indicando que tales ítems realizan un aporte superior al 50 \% de su variabilidad a la explicación de las variables latentes a las que se encuentran asociadas (Schreiber et al., 2006).

\section{Discusión}

El presente estudio contribuye a la evaluación del fenómeno de la autotrascendencia en el contexto local. La técnica propuesta favorecería el incremento de los estudios en el área de la psicología de la religión en Argentina. El Inventario de Autotrascendencia para Adultos (ASTI) (Levenson et al., 2005) ha demostrado propiedades psicométricas adecuadas, lo que posibilitaría su administración. El mismo presenta 18 ítems similares a los elaborados por Levenson et al., (2005). La consistencia interna resulta similar a la reportada por los autores en la validación original de la técnica (0,64 s $a \leq 0,75)$. Si bien el factor alienación ha presentado una confiabilidad menor al de autotrascendencia, esto puede explicarse porque la faceta se encuentra integrada por una menor cantidad de ítems (Martínez-Arias, Hernández, y Hernández, 2006; Raykov y Marcoulides, 2008, 2011). Por su parte, el AFC brinda apoyo empírico para el modelo bifactorial de la ASTI, presentando un ajuste adecuado a los datos obtenidos en la población estudiada. Estos resultados apoyan los reportados en el contexto internacional (Beaumont, 2009; Michael Dambrun, 2017; Ireland, 2013; Koller et al., 2017; Roháriková et al., 2013). Por otra parte, se observa que los parámetros estimados alcanzan valores óptimos ( $r \quad 0,70$ ) (Schreiber et al., 2006). Esto estaría indicando que los reactivos que integran cada dimensión realizan un aporte superior al 50 \% de su variabilidad a la explicación de la dimensión a la cual reporta cada uno de ellos. De esta manera, es posible afirmar que todos los elementos son representativos de las dimensiones a las cuales pertenecen. Esta característica resulta importante ya que todos los ítems parecen tener una relevancia similar respecto del modelo. Esto estaría dando cuenta de una estructura interna del instrumento parsimoniosa en la que no existe multicolinealidad entre los ítems explicando cada uno de ellos una parte importante del constructo en cuestión.

Cabe señalar, sin embargo, que en el presente artículo se presentan principalmente resultados acerca de la estructura factorial (validez factorial) y de la consistencia interna (fiabilidad) de la ASTI, lo cual supone un primer abordaje, de naturaleza exploratoria, en la tarea de adaptar y testear en el contexto local el instrumento. Por 
este motivo, futuros estudios deberían continuar explorando las propiedades psicométricas de la ASTI en Argentina.

Es necesario considerar, asimismo, en cuanto a las limitaciones muestrales, que el trabajo emplea exclusivamente población de estudiantes de nivel medio y superior. La literatura especializada ha sugerido que, en psicología de la religión, resulta de amplia relevancia explorar las propiedades psicométricas de las técnicas empleadas en población religiosa (Hill, 2012; Kapuscinski y Masters, 2010; Simkin, 2017b). Por este motivo, se sugiere en próximos estudios interesados en la evaluación de los constructos numinosos considerar explorar las propiedades de la ASTI en este tipo de población.

\section{Referencias}

Ai, A. L., Wink, P., Gall, T. L., Dillon, M. and Tice, T. N. (2017). Assessing Reverence in Contexts. Journal of Humanistic Psychology, 57(1), 64-97. doi: https://doi.org/10.1177/0022167815586657

Allport, G. W. and Ross, J. M. (1967). Personal religious orientation and prejudice. Journal of Personality and Social Psychology, 5(4), 432-443. doi: https://doi.org/10.1037/h0021212

Beaumont, S. L. (2009). Identity Processing and Personal Wisdom: An Information-Oriented Identity Style Predicts Self-Actualization and Self-Transcendence. Identity, 9(2), 95-115. doi: https:// doi.org/10.1080/15283480802669101

Bentler, P. M. (1990). Comparative fit indexes in structural models. Psychological Bulletin, 107(2), 238-246. doi: https://doi.org/10.1037/0033-2909.107.2.238

Boyatzis, C. J. (2009). Examining Religious and Spiritual Development During Childhood and Adolescence. In M. deSouza, L. J. Francis, J. O'Higgins-Norman and D. Scott. (Eds.), International Handbook of Education for Spirituality, Care and Wellbeing (pp. 51-69). Dordrecht: Springer.

Choi, N. G. and Landeros, C. (2011). Wisdom from Life's Challenges: Qualitative Interviews with Low- and Moderate-Income Older Adults Who Were Nominated as Being Wise. Journal of Gerontological Social Work, 54(6), 592-614. doi: https://doi.org/10.1080/01634372.20 11.585438

Dambrun, M. (2017). Self-centeredness and selflessness: happiness correlates and mediating psychological processes. Peer J., 5(3), e3306. doi: https://doi.org/10.7717/peerj.3306 
Dambrun, M., y Ricard, M. (2012). La transcendance de soi et le bonheur: une mise à l'épreuve du modèle du bonheur basé sur le soi centré-décentré. Les Cahiers Internationaux de Psychologie Sociale, 93(1), 89. doi: https://doi.org/10.3917/cips.093.0089

Dominguez-Lara, S. (2018). Fiabilidad y alfa ordinal. Actas Urológicas Españolas, 42(2), 140-141. doi: https://doi.org/10.1016/j.acuro.2017.07.002

Duriez, B., Fontaine, J. R. J., Hutsebaut, D. and Leuven, K. U. (2000). A further elaboration of the post-critical belief scale: Evidence for the existence of four different approaches to religion in flanders-belgium. Psychologica Belgica, 40(1967), 153-181.

Espinoza, S. C. y Novoa-Muñoz, F. (2018). Ventajas del alfa ordinal respecto al alfa de Cronbach ilustradas con la encuesta AUDIT-OMS. Rev Panam Salud Publica Pan American Journal of Public Health, 42, e65. doi: https://doi.org/10.26633/RPSP.2018.65

Garcia-Romeu, A. (2010). Self-transcendence as a measurable transpersonal construct. Journal of Transpersonal Psychology, 42(1), 26-47.

Glück, J., König, S., Naschenweng, K., Redzanowski, U., Dorner, L., Straßer, I. and Wiedermann, W. (2013). How to measure wisdom: content, reliability, and validity of five measures. Frontiers in Psychology, 4(JUL), 1-13. doi: https://doi.org/10.3389/fpsyg.2013.00405

González-Rivera, J. A. y Pagán Torres, O. M. (2018). Desarrollo y validación de un instrumento para medir estrategias de afrontamiento religioso. Revista Evaluar, 18(1), 70-86.

Greene, D. C., Britton, P. J. and Shepherd, J. B. (2016). LGBTQ Aging: Mental Health at Midlife and Older Adulthood. Journal of LGBT Issues in Counseling, 10(4), 180-196. doi: https://doi.org/ $10.1080 / 15538605.2016 .1233839$

Hair, J. F., Black, W. C., Babin, B. J. and Anderson, R. E. (2009). Multivariate Data Analysis. London, UK: Prentice Hall.

Heo, M., Kim, N. and Faith, M. S. (2015). Statistical power as a function of Cronbach alpha of instrument questionnaire items. BMC Medical Research Methodology, 15(1), 86. doi: https://doi. org/10.1186/s12874-015-0070-6

Hill, P. C. (2012). Measurement Assessment and Issues in the Psychology of Religion and Spirituality. In R. F. Paloutzian and C. L. Park (Eds.), Handbook of Psychology of Religion and Spirituality (pp. 48-75). New York, USA: The Guilford Press. 
Hu, L. and Bentler, P. M. (1999). Cutoff criteria for fit indexes in covariance structure analysis: Conventional criteria versus new alternatives. Structural Equation Modeling: A Multidisciplinary Journal, 6(1), 1-55. doi: https://doi.org/10.1080/10705519909540118

Ireland, M. J. (2013). Meditation and Psychological Health: Modeling Theoretically Derived Predictors, Processes, and Outcomes. Mindfulness, 4(4), 362-374. doi: https://doi.org/10.1007/ s12671-012-0136-z

Jackson, D., Gillaspy, J. and Purc-Stephenson, R. (2009). Reporting practices in confirmatory factor analysis: an overview and some recommendations. Psychological Methods, 14(1), 6-23. doi: https://doi.org/10.1037/a0014694

Jennings, P. A., Aldwin, C. M., Levenson, M. R., Spiro, A. and Mroczek, D. K. (2006). Combat Exposure, Perceived Benefits of Military Service, and Wisdom in Later Life: Findings from the Normative AgingStudy.ResearchonAging,28(1),115-134.doi:https://doi.org/10.1177/0164027505281549

Kapuscinski, A. N. and Masters, K. S. (2010). The current status of measures of spirituality: A critical review of scale development. Psychology of Religion and Spirituality, 2(4), 191-205. doi: https://doi.org/10.1037/a0020498

Kim, S. and Knight, B. G. (2015). Adaptation of the three-dimensional wisdom scale (3D-WS) for the Korean cultural context. International Psychogeriatrics, 27(2), 267-278. doi: https://doi. $\operatorname{org} / 10.1017 /$ S1041610214002178

Koller, I., Levenson, M. R. and Glück, J. (2017). What do you think you are measuring? A mixed-methods procedure for assessing the content validity of test items and theory-based scaling. Frontiers in Psychology, 8(February). doi: https://doi.org/10.3389/fpsyg.2017.00126

Konig, S. and Gluck, J. (2014). "Gratitude Is With Me All the Time": How Gratitude Relates to Wisdom. The Journals of Gerontology Series B: Psychological Sciences and Social Sciences, 69(5), 655-666. doi: https://doi.org/10.1093/geronb/gbt123

König, S. and Glück, J. (2013). Individual Differences in Wisdom Conceptions: Relationships to Gratitude and Wisdom. The International Journal of Aging and Human Development, 77(2), 127-147. doi: https://doi.org/10.2190/AG.77.2.c

Le, T. N. (2008). Age differences in spirituality, mystical experiences and wisdom. Ageing and Society, 28(03), 383-411. doi: https://doi.org/10.1017/S0144686X0700685X 
Le, T. N. (2011). Life Satisfaction, Openness Value, Self-Transcendence, and Wisdom. Journal of Happiness Studies, 12(2), 171-182. doi: https://doi.org/10.1007/s10902-010-9182-1

Le, T. N. and Levenson, M. R. (2005). Wisdom as self-transcendence: What's love (yamp; individualism) got to do with it? Journal of Research in Personality, 39(4), 443-457. doi: https://doi. org/10.1016/j.jrp.2004.05.003

Lee, S., Choun, S., Aldwin, C. M. and Levenson, M. R. (2015). Cross-Cultural Comparison of SelfTranscendent Wisdom Between the United States and Korea. Journal of Cross-Cultural Gerontology, 30(2), 143-161. doi: https://doi.org/10.1007/s10823-015-9259-8

Levenson, M. R., Aldwin, C. M. and Igarashi, H. (2013). Religious development from adolescence to middle adulthood. In R. F. Paloutzian y C. L. Park (Eds.), Handbook of the psychology of religion and spirituality (pp. 183-198). New York, USA: The Guilford Press.

Levenson, M. R., Jennings, P. A., Aldwin, C. M. and Shiraishi, R. W. (2005). Self-transcendence: conceptualization and measurement. International Journal of Aging y Human Development, 60(2), 127-143. doi: https://doi.org/10.2190/XRXM-FYRA-7UOX-GRCO

Martínez-Arias, M. R., Hernández, M. J. y Hernández, M. V. (2006). Psicometría. Madrid, España: Alianza Editorial.

Mezzadra, J. y Simkin, H. (2017). Validación de la Escala Abreviada de Afrontamiento Religioso Brief-RCOPE en el Contexto Argentino en estudiantes de confesión católica. Evaluar, 17(1), $18-28$.

Morledge, T. J., Allexandre, D., Fox, E., Fu, A. Z., Higashi, M. K., Kruzikas, D. T. and Reese, P. R. (2013). Feasibility of an Online Mindfulness Program for Stress Management-A Randomized, Controlled Trial. Annals of Behavioral Medicine, 46(2), 137-148. doi: https://doi.org/10.1007/ s12160-013-9490-x

Muñiz, J., Elosua, P. y Hambleton, R. K. (2013). Directrices para la traducción y adaptación de los tests: Segunda edición. Psicothema, 25(2), 151-157. doi: https://doi.org/10.7334/ psicothema2013.24

Pargament, K. I., Smith, B. W., Koenig, H. G. and Perez, L. (1998). Patterns of Positive and Negative Religious Coping with Major Life Stressors. Journal for the Scientific Study of Religion, 37(4), 710. doi: https://doi.org/10.2307/1388152 
Piedmont, R. L. (1999). Does Spirituality Represent the Sixth Factor of Personality? Spiritual Transcendence and the Five-Factor Model. Journal of Personality, 67(6), 985-1013. doi: https://doi.org/10.1111/1467-6494.00080

Piedmont, R. L. (2004). Assessment of spirituality and religious sentiments, technical manual. Timonium, Maryland: (n.d.)

Rabbia, H. H., Brussino, S. y Vaggione, J. M. (2012). Escala de Creencias Post-Críticas: análisis psicométrico para su aplicación al estudio de la religiosidad en Argentina. Revista de Psicología, 8(16), 85-109.

Rajani, F. (2015). Theory of Gerotranscendence: an Analysis. European Psychiatry, 30, 1467. doi: https://doi.org/10.1016/S0924-9338(15)31138-X

Raykov, T. and Marcoulides, G. A. (2008). An Introduction to Applied Multivariate Analysis. New York, USA: Taylor \& Francis.

Raykov, T. and Marcoulides, G. A. (2011). Introduction to Psychometric Theory. New York, USA: Taylor \& Francis.

Reed, P. G. (1991). Self-Transcendence and Mental Health in Oldest-Old Adults. Nursing Research, 40(1), 5-11. doi: https://doi.org/10.1097/00006199-199101000-00002

Rivera-Ledesma, A., Zavala-Jimenez, S., Montero-Lopez Lena, M. and Maria Garcia-Mendez, R. (2016). Validation of the Age Universal I-E Scale in Mexican Subjects. Universitas Psychologica, 15(4). doi: https://doi.org/10.1114/Javeriana.upsy15-4.vaus

Roháriková, V., Špajdel, M., Cviková, V. and Jagla, F. (2013). Tracing the relationship between wisdom and health. Activitas Nervosa Superior Rediviva, 55(3), 95-102.

Schermelleh-Engel, K., Moosbrugger, H. and Müller, H. (2003). Evaluating the Fit of Structural Equation Models: Tests of Significance and Descriptive Goodness-of-Fit Measures. Methods of Psychological Research Online, 8(2), 23-74.

Schreiber, J. B., Nora, A., Stage, F. K., Barlow, E. A. and King, J. (2006). Reporting Structural Equation Modeling and Confirmatory Factor Analysis Results: A Review. The Journal of Educational Research, 99(6), 323-338. doi: https://doi.org/10.3200/JOER.99.6.323-338

Schumacker, R. E. and Lomax, R. G. (2004). A beginner's guide to estructural equation modeling. New Jersey, USA: Lawrence Erlbaum Associates. 
Simkin, H. (2017a). Adaptación y Validación al Español de la Escala de Evaluación de Espiritualidad y Sentimientos Religiosos (ASPIRES): la trascendencia espiritual en el modelo de los cinco factores. Universitas Psychologica, 16(2), 1-12. doi: https://doi.org/10.11144/Javeriana. upsy16-2.aeee

Simkin, H. (2017b). Psicología de la Religión: adaptación y validación de escalas de evaluación psicológica en el contexto argentino. Anuario de Investigaciones, 24, 177-185.

Simkin, H. y Etchezahar, E. (2013). Las orientaciones religiosas extrínseca e intrínseca: validación de la "Age Universal" I-E Scale. Psykhe, 22(1), 97-106. doi: https://doi.org/10.7764/ psykhe.22.1.477

Simkin, H. and Piedmont, R. L. (2018). Adaptation and Validation of the Assessment of Spirituality and Religious Sentiments (ASPIRES) scale short form into spanish. Latinoamerican Journal of Positive Psychology, 4, 97-107.

Tornstam, L. (1994). Gero-transcendence: A theoretical and empirical exploration. In L. E. Thomas and S. A. Eisenhandler. (Eds.), Aging and the religious dimension (pp. 203-225). London, UK: Auburn House.

Ventura, J. L. (2018). ¿Es el final del alfa de Cronbach? Adicciones, 20(10), 1-2. doi: https://doi. org/10.13140/RG.2.2.25157.55529

Ventura León, J. L. y Caycho Rodriguez, T. (2017). El coeficiente Omega: un método alternativo para la estimación de la confiabilidad. Revista Latinoamericana de Ciencias Sociales, Niñez y Juventud, 15(1), 625-627.

Vieten, C., Estrada, M., Cohen, A. B., Radin, D., Schlitz, M. M. and Delorme, A. (2014). Engagement in a community-based integral practice program enhances well-being. International Journal of Transpersonal Studies, 33(2), 1-15.

Viladrich, C., Angulo-Brunet, A. y Doval, E. (2017). Un viaje alrededor de alfa y omega para estimar la fiabilidad de consistencia interna. Anales de Psicología, 33(3), 755. doi: https://doi. org/10.6018/analesps.33.3.268401

Zhang, Z. and Yuan, K. (2016). Robust Coefficients Alpha and Omega and Confidence Intervals With Outlying Observations and Missing Data. Educational and Psychological Measurement, 76(3), 387-411. doi: https://doi.org/10.1177/0013164415594658 
Zinbarg, R. E., Revelle, W., Yovel, I. and Li, W. (2005). Cronbach's $\alpha$, Revelle's $\beta$, and Mcdonald's $\omega \mathrm{H}$ : their relations with each other and two alternative conceptualizations of reliability. Psychometrika, 70(1), 123-133. doi: https://doi.org/10.1007/s11336-003-0974-7 